\title{
LA FAMILIA ARANEIDAE (ARACHNIDA: ARANEAE) EN LA COLECCIÓN DEL MUSEO NACIONAL DE HISTORIA NATURAL DE SANTO DOMINGO Y DOS NUEVOS REGISTROS PARA LA HISPANIOLA
}

\author{
Gabriel de los $\operatorname{Santos}^{1}$ y Solanlly Carrero Jiménez ${ }^{2}$ \\ Museo Nacional de Historia Natural de Santo Domingo (MNHNSD). Calle César Nicolás Penson, \\ Plaza de la Cultura, Santo Domingo, República Dominicana. \\ 1'g.delossantos@museohistorianatural.gov.do, ${ }^{2}$ s.carrero@museohistorianatural.gov.do
}

\section{RESUMEN}

Se revisó la colección de Araneidae depositada en el Museo Nacional de Historia Natural de Santo Domingo (MNHNSD). Esta consta de 894 especímenes, distribuidos en 22 géneros y 34 especies. Acanthepeira venusta (Banks, 1896) y Metazygia genialis (Keyserling, 1892) se registran por primera vez para la República Dominicana y La Hispaniola. La colección incluye dos paratipos correspondientes a Aculepeira busu Levi, 1991 y Wagneriana vegas Levi, 1991. Se presenta el catálogo de la colección.

Palabras clave: Araneidae, La Hispaniola, República Dominicana, catálogo, nuevos registros.

\section{ABSTRACT}

The Araneidae collection from the Museo Nacional de Historia Natural de Santo Domingo (MNHNSD) is reviewed. The collection holds 894 specimens, distributed in 22 genera and 34 species. Acanthepeira venusta (Banks, 1896) and Metazygia genialis (Keyserling, 1892) are recorded for the first time from Dominican Republic and the island of Hispaniola. This collection includes two paratypes corresponding to Aculepeira busu Levi, 1991 and Wagneriana vegas Levi, 1991. A catalog of the collection is presented.

Key words: Araneidae, Hispaniola, Dominican Republic, catalog, new records.

\section{INTRODUCCIÓN}

La familia Araneidae ocupa el tercer lugar dentro del Orden Araneae en cuanto a diversidad, con 3,006 especies a nivel mundial, distribuidas en 168 géneros (Platnick, 2011). En La Hispaniola (República Dominicana y Haití), ocupa el primer lugar con 65 especies (58 actuales y siete fósiles), repartidas en 30 géneros (26 actuales y cuatro fósiles), de acuerdo con Perez-Gelabert (2008). Esta familia está distribuida en todo el planeta y algunas especies son colonizadoras de áreas aisladas (Levi, 2005).

Bryant (1945 y 1948) hace la mayor contribución al conocimiento de esta familia en La Hispaniola, describiendo y registrando un total de 31 especies. En 1988, Wunderlich describió seis de las siete especies fósiles que se conocen de esta familia. Periódicamente, Levi (1991a, 1991b, 1992, 1995 y 1999), describió y registró unas 11 especies; ya en el siglo XXI, Piel (2001), registra 4 especies del género Metepeira Cambridge, 1903. En el año 2002, Penney y Perez-Gelabert registran otras 10 especies y Alayón García (2007) describe una nueva especie del género Pozonia Schenkel, 1953 (en Perez-Gelabert, 2008). Otros registros corresponden a Archer (1958), Chickering (1964) y Berman y Levi (1971).

Este artículo lleva como propósito dar a conocer el catálogo de esta familia, el cual es parte de los resultados del proceso de organización y catalogación de la colección de arácnidos del 
MNHNSD, iniciado en el año 2007 con la colaboración de Alexander Sánchez-Ruiz, de Cuba, y Kennida Polanco, de República Dominicana.

\section{OBJETIVOS}

- Analizar la composición de la familia Araneidae en la colección de arácnidos del MNHNSD y hacer su actualización taxonómica.

- Presentar dos nuevos registros de especies de esta familia para La Hispaniola.

\section{MATERIALES Y MÉTODOS}

Se examinaron todos los especímenes de la familia Araneidae depositados en la colección aracnológica del MNHNSD. Se actualizó la información taxonómica y se determinaron algunos especímenes. Para la determinación de los especímenes se siguió a Levi (2002 y 2005) y a Platnick (2011) para la actualización taxonómica.

Con excepción de Allocyclosa sp., Araneus sp., Mecynogea sp. y Pozonia sp., las demás especies determinadas hasta género (Tabla 1) no están incluidas en el total de 34 especies.

\section{RESULTADOS Y DISCUSIÓN}

La colección del MNHNSD consta de 353 lotes con un total de 894 especímenes, distribuidos en 22 géneros y 34 especies. Estos números representan el 84\% y 59\%, respectivamente, de los registros conocidos para la isla. Esta familia también es la más numerosa dentro de la colección de arañas. Unos 56 lotes, conteniendo 156 especímenes, aún restan por ser determinados.

Respecto a lo registrado para la isla, tomando en cuenta aquellos géneros con más de dos (2) especies registradas, los géneros mejor representados en la colección son Metazygia (6 especies), Metepeira (4) y Micrathena (4). Ver anexo. Los géneros Eustala (2) y Neoscona (3) están pobremente representados.

En cuanto a la cantidad de lotes y especímenes, Gasteracantha cancriformis (Linnaeus, 1758) supera por mucho a las demás especies con 67 lotes y 256 especímenes (Anexo). A esta le siguen Neoscona sp., con 31 lotes y 62 especímenes, Micrathena militaris (Fabricius, 1775), con 21 lotes y 44 especímenes, y Argiope argentata (Fabricius, 1775), con 19 lotes y 41 especímenes.

Gasteracantha es el género con mayor porcentaje de especímenes 34\% (fig. 1). A este le siguen: Metepeira, con un 12\%, Argiope y Neoscona, 10\%, y Micrathena, con un 9.5\%. Los géneros Aculepeira, Allocyclosa, Araneus, Larinia, Ocrepeira, Pozonia, Wagneriana y Witica, comprenden menos del $2 \%$ de los especímenes.

Los especímenes de Aculepeira busu Levi, 1991 y Wagneriana vegas Levi, 1991, corresponden a paratipos (uno de cada especie). Las especies Acanthepeira venusta (Banks, 1896) y Metazygia genialis (Keyserling, 1892) se registran por primera vez para la República Dominicana y La Hispaniola.

Los aracnólogos Hebert W. Levi, Laura Leibensperger, William H. Piel, Giraldo Alayón y Alexander Sánchez-Ruiz aparecen registrados como especialistas que han realizado determinaciones de los especímenes de esta colección. Entre los principales colectores figuran Eugenio de Jesús Marcano, Félix Del Monte y Kelvin Guerrero. El registro más antiguo de la 
colección es de Metazygia pallidula (Keyserling, 1864), colectado en Panamá en el año 1939 por Arthur M. Chickering. Las regiones de la República Dominicana mejor representadas son la Este (Parque Nacional del Este) y la Suroeste (provincias Pedernales e Independencia).

Los resultados obtenidos en este trabajo nos permiten conocer el buen estado que presenta esta familia en la colección nacional de referencia. En la República Dominicana nunca se ha realizado ningún proyecto (con recursos nacionales) enfocado exclusivamente al estudio de los arácnidos; por eso no sorprende que la colección de arañas cuente con poco más de 1,800 lotes, los cuales han llegado de manera lateral como resultado de proyectos y viajes de campo enfocados en otros grupos. Además, los especialistas extranjeros que visitan el país se marchan sin dejar réplicas en el MNHNSD de los especímenes colectados; por esta razón, tampoco sorprende que esta colección (Araneidae) solo cuente con dos ejemplares tipo. La falta de aracnólogos nacionales dedicados a tiempo completo a este trabajo ha sido un factor de mucho peso en la problemática descrita.

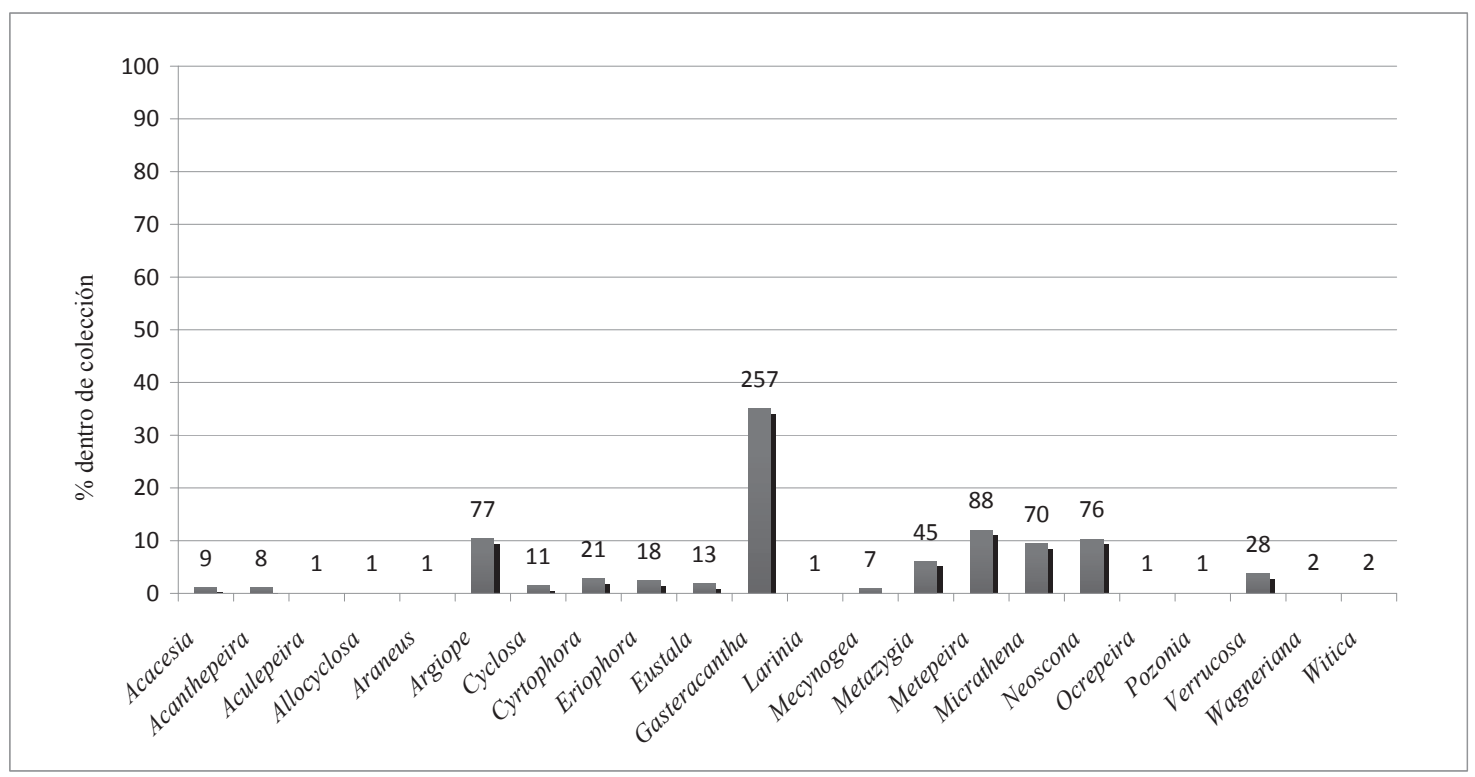

Figura 1. Relación de la cantidad de especímenes por género dentro de la colección de Araneidae.

\section{ACANTHEPEIRAVENUSTA(BANKS, 1896) YMETAZYGIAGENIALIS(KEYSERLING, 1892): DOS NUEVOS REGISTROS PARA LA HISPANIOLA}

Aunque en la colección solo está representado el 59\% de los registros conocidos para la isla, los nuevos registros de las especies, Acanthepeira venusta (Banks, 1896) y Metazygia genialis (Keyserling, 1892), encontrados en este trabajo nos dicen que todavía queda mucho por conocer de este interesante grupo.

Acanthepeira venusta se conoce de Estados Unidos y Cuba (Bryant, 1949; Levi, 1976; Alayón García, 2000), mientras que Metazygia genialis, de Brasil (Levi, 1995). El caso de A. venusta no sorprende demasiado debido a la cercanía de Cuba con La Hispaniola. Pero el caso de $M$. genialis sí resulta interesante y nos permite conocer más sobre la influencia antropogénica en la distribución/dispersión de las especies, esto así, porque la única vía que consideramos para la dispersión en esta nueva localidad es la introducción por medios de transporte humano. 


\section{LITERATURA CITADA}

Alayón García, G. 2000. Las arañas endémicas de Cuba (Arachnida: Araneae). Revista Ibérica Aracnología 2, 1-48.

Alayón García, G. 2007. Especie nueva de Pozonia (Araneae: Araneidae) para República Dominicana. Solenodon, 6, 41-44.

Archer, A. F. 1958. Studies in the orbweaving spiders (Argiopidae) 4. American Museum Novitates, 1922, 1-21.

Berman, J. D. y H. W. Levi. 1971. The orb-weaver genus Neoscona in North America (Araneae: Araneidae). Bulletin of the Museum of Comparative Zoology, 141 (8), 465-500.

Bryant, E. B. 1945 The Argiopidae of Hispaniola. Bulletin of the Museum of Comparative Zoology, 95, 357-418.

Bryant, E. B. 1948. The Spiders of Hispaniola. Bulletin of the Museum of Comparative Zoology, $100,332-447$.

Bryant, E. B. 1949. Acanthepeira venusta (Banks) (Araneae). Psyche, 56, 175-179.

Chickering, A. M. 1964. The genus Micrathena (Araneae, Araneidae) in the West Indies. Bulletin of the Museum of Comparative Zoology, 131 (8), 251-281.

Levi, H. W. 1976. The orb-weaver genera Verrucosa, Acanthepeira, Wagneriana, Acacesia, Wixia, Scoloderus and Alpaida north of Mexico. Bulletin of the Museum of Comparative Zoology, 147, 351-391.

Levi, H. W. 1991a. The Neotropical and Mexican species of the orb-weaver genera Araneus, Dubiepeira new genus, and Aculepeira (Araneae: Araneidae). Bulletin of the Museum of Comparative Zoology, 152, 167-315.

Levi, H. W. 1991b. The Neotropical orb-weaver genera Edricus and Wagneriana (Araneae: Araneidae). Bulletin of the Museum of Comparative Zoology, 152, 363-415.

Levi, H. W. 1992. American Neoscona and corrections to previous revisions of Neotropical orbweavers (Araneae: Araneidae). Psyche, 99, 221-239.

Levi, H. W. 1995. The Neotropical orb-weaver genus Metazygia (Araneae: Araneidae). Bulletin of the Museum of Comparative Zoology, 154, 63-151.

Levi, H. W. 1999. The Neotropical and Mexican orb weavers of the genera Cyclosa and Allocyclosa (Araneae: Araneidae). Bulletin of the Museum of Comparative Zoology, 155, 299-379.

Levi, H. W. 2002. Keys to the genera of araneids orbweavers (Araneae, Araneidae) of the Americas. The Journal of Arachnology, 30, 527-562.

Levi, H. W. 2005. Araneidae. p. 68 en D. Ubick, P. Paquin, P.E. Cushing, y V. Roth (eds.) Spiders of North America: an identification manual. American Arachnological Society.

Penney, D. y Perez-Gelabert, D. E. 2002. Comparison of the Recent and Miocene Hispaniolan spider faunas. Revista Ibérica de Aracnología, 6, 203-223.

Perez-Gelabert, D. E. 2008. Arthropods of Hispaniola (Dominican Republic and Haiti): A checklist and bibliography. Zootaxa, 1831, 1-530. 
Piel, W. H. 2001. The systematics of Neotropical orb-weaving spiders in the genus Metepeira (Araneae: Araneidae). Bulletin of the Museum of Comparative Zoology, 157, 1-92.

Platnick, N. I. 2011. The world spider catalog, version 11.5. American Museum of Natural History, (en línea): http://research.amnh.org/iz/spiders/catalog/COUNTS.html 15.II.2011.

Wunderlich, J. 1988. Die Fossilen Spinnen im Dominikanischem Bernstein. Beiträge zur Araneologie, 2, 378 pp.

Anexo. Catálogo de la colección de Araneidae (Arachnida: Araneae) del Museo Nacional de Historia Natural de Santo Domingo (MNHNSD).

Acacesia Simon, 1895

Acacesia hamata (Hentz, 1847) (4 lotes, 4 especímenes)

1 MNHNSD 09.31, prov. Peravia, Baní, Honduras, El Matadero. 25-26.X.2008, V. De La Rosa, A. Hilario, G. de los Santos. 19 MNHNSD 09.32, prov. La Romana, Isla Saona. 1 9 MNHNSD 09.33, prov. Pedernales, bosque transición Aceitillar-Cabo Rojo. 19.I.2002, S. Medrano. 1q MNHNSD 09.35, prov. Pedernales, Cabo Rojo. 20.V.1992, K. Guerrero.

Acacesia sp. (4 lotes, 5 especímenes)

1 juv MNHNSD 09.36, prov. San Juan, La Guácara, Pico Duarte. 08.VII.1992, M. Ivie, K. Guerrero. 2 juv MNHNSD 09.37, prov. Pedernales, Las Abejas, Sierra de Bahoruco. Bajo tronco. 15.VIII.1991, K. Guerrero. 1 juv MNHNSD 09.402, prov. Santiago, Tamboril. 4.XI.1979, E. Marcano. 1 ㅇ MNHNSD 09.295, prov. Peravia, Baní, Honduras, El Matadero. 25-26.X.2008. V. De la Rosa, A. Hilario, G. de los Santos.

Acanthepeira Marx, 1883

Acanthepeira venusta (Banks, 1896) (3 lotes, 7 especímenes)

2 早 MNHNSD 09.38, prov. Independencia, La Descubierta, Los Borbollones. 30.I.1991, F. Del Monte. 2 ㅇ $10^{\lambda}$ MNHNSD 09.39, prov. Duarte, San Francisco de Macorís, La Boca. 04.X.1980, B. Reynoso. 2 9 MNHNSD 09.40, prov. Duarte, San Francisco de Macorís, La Boca. 05.IV.1980, B. Reynoso.

Acanthepeira sp. (1 lote, 1 espécimen)

1 juv MNHNSD 09.41, prov. Duarte, San Francisco de Macorís, La Boca. 21.VI.1980, B. Reynoso.

Aculepeira Chamberlin \& Ivie, 1942

Aculepeira busu Levi, 1991 (1 lote, 1 espécimen)

PARATIPO: 1 ㅇ MNHNSD 09.42, prov. Pedernales, in gorge nr. Isla, nr. Hoyo de Pelempito. 10.XII.1978, D. G. Robinson.

Allocyclosa Levi, 1999

Allocyclosa sp. (1 lote, 1 espécimen)

1 P MNHND 09.1183, prov. Peravia, Baní, La Montería, El Manaclar, loma Los Guayuyos, UTM 19Q 354040m.E 2036965m.N. 29.VIII.2009, G. de los Santos.

Araneus Clerck, 1757

Araneus sp. (1 lote, 1 espécimen)

1 우 MNHNSD 09.53, prov. Santiago, Tamboril, Licey Al Medio. 29.IX.1979, E. Marcano.

Argiope Audouin, 1827

Argiope argentata (Fabricius, 1775) (19 lotes, 41 especímenes)

2 ㅇ MNHNSD 09.56, prov. La Altagracia, San Rafael del Yuma, La Gran Chorra. 14.III.1992. 10 ㅇ MNHNSD 09.57, prov. Independencia, La Descubierta, Isla Cabritos. 04.VII.1991, E. Marcano. 3 q MNHNSD 09.58, prov. Azua, Tábara Abajo, Río Arroyo Blanco. 22.XII.1979, E. Marcano. 1 ㅇ MNHNSD 09.59, prov. Independencia, La Descubirta, Isla 
Cabritos. 27.IV.2007, K. Polanco. 19 MNHNSD 09.60, prov. Independencia, La Descubirta, Isla Cabritos, La Playita. D. G. Robinson. 1 q MNHNSD 09.61, prov. Distrito Nacional, Santo Domingo, Miramar. 16.II.1979, Salcedo. 1 ㅇ MNHNSD 09.62, prov. Distrito Nacional, Santo Domingo. 03.X.1976, G. Gómez. 1 q 1 juv MNHNSD 09.63, prov. Independencia, La Descubierta, Isla Cabritos. 01.VIII.1981, E. Marcano. 2 9 MNHNSD 09.64, prov. La Altagracia, Higüey, El Salado, Verón. 10.VIII.1979, E. Marcano. 1 NNNSD 09.65, prov. Independencia, Jimaní, $2 \mathrm{~km}$ SE del Limón. 30.I.1991, F. Del Monte. 2 q MNHNSD 09.72, prov. Puerto Plata, Luperón, La Isabela. 04.X.1981, E. Marcano. 19 MNHNSD 09.74, prov. Pedernales, Cabo Rojo. 20.V.1992. 1 q MNHNSD 09.73, prov. Peravia, Baní,

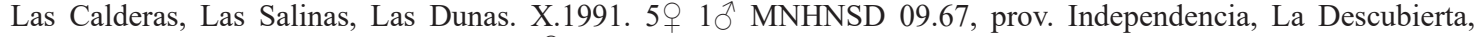
Isla Cabritos. 18.VI.1981, E. Marcano. 1 ㅇ MNHNSD 09.68, prov. Santo Domingo, Boca Chica. 06.I.1977, J. A. Ottenwalder. 3 + MNHNSD 09.69, prov. Azua, Hatillo, Las Charcas. 22.XII.1979, E. Marcano. 1ㅇ MNHNSD 09.70, prov. Pedernales, Pedernales, Las Mercedes, carretera ALCOA. 24.I.1981, J. A. Ottenwalder, S. Incháustegui, M. G.

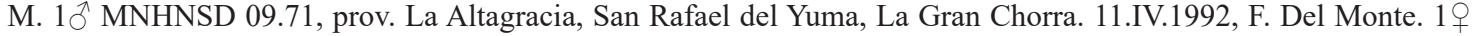
MNHNSD 09.1185, prov. Peravia, Baní, Honduras, loma Las Yayas, UTM 19Q 349418m.E 2035185m.N. 2.VIII.2009, G. de los Santos.

Argiope trifasciata (Forskål, 1775) (12 lotes, 25 especímenes)

1 ㅇ MNHNSD 09.75, prov. Monseñor Nouel, Bonao, Casabito. 25.VIII.1979, E. Marcano. 19 MNHNSD 09.76, prov. La Altagracia, Las Lagunas de Nisibón, Las Cañas. 29.III.1980, E. Marcano. 1 우 MNHNSD 09.77, prov. Distrito Nacional, Santo Domingo. K. Gómez. 1 ㅇ MNHNSD 09.78, prov. Monseñor Nouel, Bonao, Tireito. K. Polanco. 1 오 MNHNSD 09.79, prov. Distrito Nacional, Santo Domingo, jardín de Ciencias de la UASD. 5.XI.1984, F. Del Monte. 1 juv. MNHNSD 09.80, prov. La Altagracia. 26.I.1980, E. Marcano. 1 우 MNHNSD 09.81, prov. Monte Plata, Sabana Grande de Boyá, Cañada Grande. 10.VIII.1985, A. Villalba. 11우 MNHNSD 09.82, María Trinidad Sánchez, Nagua, Boca del Gran Estero. 26.VIII.1980, E. Marcano. 19 MNHNSD 09.1184, prov. La Vega, Constanza, Cuevita, $18^{\circ}$ 44.469’ N 70 40.227’ W. 28.XI.2002, D. Perez, B. Hierro, R. Bastardo. 2 9 MNHNSD 09.364, Isla Navassa. 6.V.1999, S. Navarro. 2 juv. MNHNSD 09.55, prov. Duarte, San Francisco de Macorís, Colón, La Boca. 01.X.1980, B. Reynoso. 2 ㅇ MNHNSD 09.87, prov. La Vega, Constanza, La Ciénaga, 1859.999’N 70³.735’W. 31.VIII.2001, S. Medrano.

Argiope sp. (5 lotes, 11 especímenes)

1 온 MNHNSD 09.83, prov. Montecristi, carretera Montecristi-El Morro. 21.XII.1991. F. Del Monte. $1 \sigma^{\lambda} 4$ juv. MNHNSD 09.84, prov. La Altagracia, Lagunas de Nisibón, Las Cañas. 29.III.1980. 1 ㅇ 1 juv. MNHNSD 09.85, prov. Santo Domingo, Santo Domingo Oeste, Engombe. 21.V.1987, Juan Rosario. 1 ㅇ MNHNSD 09.86, Isla Navassa. 5.V.1999, S. Navarro. 2 + MNHNSD 09.365, Isla Navassa, 8.V.1999, S. Navarro.

\section{Cyclosa Menge, 1866}

Cyclosa bifurcata (Walckenaer, 1842) (1 lote, 1 espécimen)

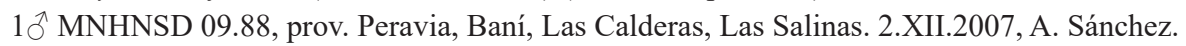

Cyclosa caroli (Hentz, 1850) (1 lote, 2 especímenes)

1 §ิ 1 ㅇ MNHNSD 09.89, prov. San Juan, Sabaneta, Alto de la Rosa. 8.I.2008, A. Sánchez, R. Ortiz.

Cyclosa walckenaeri (O.P.-Cambridge, 1889) (4 lotes, 8 especímenes)

2 q MNHNSD 09.91, prov. Pedernales, Oviedo, Oviedo Viejo. 30.XII.1984, R. Briones. 2 q MNHNSD 09.92, carretera La Romana-Higüey, 7km W represa L. Charón. 9.V.1991, K. Guerrero. 3 + MNHNSD 09.93, prov. La Altagracia, San Rafael del Yuma, Boca de Yuma. 8.V.1988, J. Infante. 1 ㅇ MNHNSD 09.94, Puerto Rico, Puente Blanco, Quebradillas. 4.V.1993.

\section{Cyrtophora Simon, 1864}

Cyrtophora citricola (Forskål, 1775) (6 lotes, 15 especímenes)

$2 \bigcirc 1$ juv. MNHNSD 09.95, prov. Azua, Las Charcas, Palmar de Ocoa. 28.I.2001, Sardis Medrano. 1ㅇ MNHNSD 09.96, prov. Independencia, La Descubierta, Isla Cabritos. 27.IV.2007, K. Polanco. 3 ㅇ MNHNSD 09.97, prov. Azua, Tábara Abajo, Las Guanabanas. 8.VII.1999, S. Medrano. 1ㅇ MNHNSD 09.98, prov. Distrito Nacional, Santo Domingo de Guzmán, Gazcue, Plaza de la Cultura Juan P. Duarte. 19.III.2001, S. Medrano. 5 q MNHNSD 09.99, prov. Azua. VII.1999, S. Medrano. 1 ㅇ 1 juv. MNHNSD 09.100, prov. San Cristóbal, San Cristóbal, Najayo. 10.X.1999, S. Medrano.

Cyrtophora sp. (2 lotes, 6 especímenes)

1 온 MNHSD 09.101, prov. Santo Domingo, Santo Domingo Oeste, Haina, Engombe. 21.XI.2007, curso UASD. 3 ำ 1 juv. MNHNSD 09.1188, prov. Peravia, Baní, La Montería, El Manaclar, loma Los Guayuyos, UTM 19Q 354040m.E 2036965m.N. 29.VIII.2009, G. de los Santos.

\section{Eriophora Simon, 1864}

Eriophora ravilla (C. L. Koch, 1844) (3 lotes, 4 especímenes)

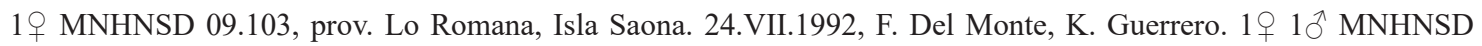


09.104, prov. Pedernales, Cabo Rojo, Isla Beata, camino a Cueva del Tubo. $17^{\circ} 36.844^{\prime} \mathrm{N} 71^{\circ} 31.379^{\prime}$ W. 3.XII.2002, D. Pérez. $1 \widehat{\jmath}$ MNHNSD 09.105, prov. Independencia, La Descubierta, Isla Cabritos. III.1991, Tammy Domíguez.

Eriophora sp. (13 lotes, 14 especímenes)

1 우 MNHNSD 09.106, prov. Santo Domingo, Santo Domingo Norte, La Victoria, Finca La Huronera. 1우 MNHNSD 09.107, prov. Peravia, Baní, Honduras, El Matadero. 23-24.XII.2007, G. de los Santos. 1 ㅇ MNHNSD 09.109, prov. Monte Plata, Bayaguana, Los Berros. 27.XII.1979, E. Marcano. 19 MNHNSD 09.110, prov. La Vega, Jarabacoa, Paso de la Perra, Nr. La Ciénaga. 15.V.2001, D. Veloz. 1 9 MNHNSD 09.111, prov. Azua, Playa de Monterío. 19.XI.1976, S. Incháustegui. 1 우 1 juv. MNHNSD 09.112, prov. San Juan, Las Matas de Farfán, Las Carreras. 27.XII.1980, E. Marcano. 1 q MNHNSD 09.113, prov. San Pedro de Macorís, Ramón Santana. 2.X.1979, Pablo Merejo. 19 MNHNSD 09.114, prov. Independencia, La Descubierta, Isla Cabritos, La Playita. 8.IV.1978, J. A. Ottenwalder, C. Sanlley, S. J. Incháustegui. 1 ㅇ MNHNSD 09.115, prov. La Altagracia, San Rafael del Yuma, Boca de Yuma. 26.III.2007, B. Farrell, K. Guerrero. 1 ㅇ MNHNSD 09.116, prov. La Altagracia, Lagunas de Nisibón, Las Cañas. 29.III.1980, E. Marcano. 1 juv. MNHNSD 09.117, prov. Independencia, La Descubierta, Isla Cabritos. 18.VI.1981, E. Marcano. 19 MNHNSD 09.350, prov. La Altagracia, $18^{\circ} 23.423^{\prime}$ N $68^{\circ} 50.453^{\prime}$ W. 31.VII.2002, D. Perez, R. Bastardo, B. Hierro. 1 ㅇ MNHNSD 09.356, prov. La Altagracia, San Rafael del Yuma, Boca de Yuma, 18²1’35”N 68³7'10”W. 26.III.2002, B. Farrell, K. Guerrero.

\section{Eustala Simon, 1895}

Eustala bisetosa Bryant, 1945 (2 lotes, 2 especímenes)

1 온 MNHND 09.1190, prov. Peravia, Baní, La Montería, El Manaclar, loma Los Pinos, UTM 19Q 352357m.E 2035657m.N. 26.IX.2009, G. de los Santos. 1 ㅇ MNHNSD 09.1191, prov. Peravia, Baní, La Montería, El Manaclar, loma Los Guayuyos, UTM 19Q 353421m.E 2036933m.N. 25.IX.2009, G. de los Santos.

Eustala sp. (6 lotes, 11 especímenes)

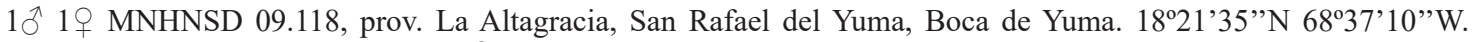
26.III.2002, B. Farrell, K. Guerrero. 1 9 MNHNSD 09.119, prov. La Altagracia. $18^{\circ} \mathrm{N} 68^{\circ} \mathrm{W} 22-31 . I I I .2002$, B. Farrell, K. Guerrero. 3 ㅇ 1juv. MNHNSD 09.120, prov. La Altagracia, San Rafael del Yuma, Boca de Yuma, 18²1'35”N 683'10"W. 26.III.2002, B. Farrell, K. Guerrero. 1ㅇ MNHNSD 09.121, prov. Independencia, La descubierta, Isla Cabritos, 6.I.1992, E. Marcano F. 1 ㅇ MNHNSD 09.1189, prov. La Altagracia, San Rafael del Yuma. 30.V.1992. 1 우

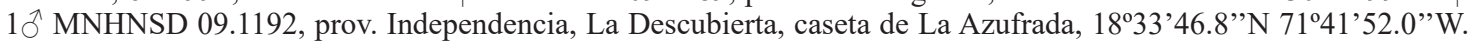
4.IX.2009, G. de los Santos.

\section{Gasteracantha Sundevall, 1833}

Gasteracantha cancriformis (Linnaeus, 1758) (67 lotes, 256 especímenes)

3 MNHNSD 09.123, prov. Peravia, Baní, Honduras, El Matadero. 23.XII.2007. 1 q MNHNSD 09.124, prov. Santo Domingo, Santo Domingo Oeste, Engombe. 21.XI.2007, curso UASD. 19 MNHNSD 09.127, prov. Pedernales, Pedernales. S. Navarro. 19 MNHNSD 09.128, prov. Samaná, Samaná. 20.XII.2007, H. Andújar. 7 ㅇ MNHNSD 09.129, prov. San José de Ocoa, $1^{\circ}$ 27.686’N 70 27.858W. 24.XI.2002, D. Pérez, B. Hierro, H. Andújar. 19 MNHNSD 09.130, prov. La Altagracia, San Rafael del Yuma, Guaraguao. 22-31.V.1992. 1 + MNHNSD 09.132, Santo Domingo, Santo Domingo Norte, La Victoria, Finca La Huronera. 3 9 MNHNSD 09.134, Isla Navassa. 5.V.1999, S. Navarro. 1 juv. MNHNSD 09.135, Peravia, Baní, Las Calderas, Las Salinas. 2.XII.2007, A. Sánchez. 2 6 juv. MNHNSD 09.201, prov. Samaná, Las Terrenas. 8.VIII.1976, D. G. Robinson, J. A. Ottenwalder. 19 MNHNSD 09.136, prov. Peravia, Baní, Honduras, El Matadero. $1^{\circ} 24.367^{\prime}$ N 70²5.703’W. 28.VII.2002, D. Pérez, R. Bastardo. 3 MNHNSD 09.137, prov. Independencia, La Descubierta, El Higüero. 30.I.1991, F. Del Monte. 1 ㅇ MNHNSD 09.139, prov. Peravia, Baní, Las Calderas, Las Salinas. X.1991. 1 \% MNHNSD 09.140, prov. Valverde, Mao, Los Quemados. 270275m.E 2155524m.N. 1.IX.2001. 19 MNHNSD 09.141, prov. Valverde, Mao, Los Quemados. 271063SE 2156344N. 1.IX.2001, K. Guerrero. 1ㅇ MNHNSD 09.143, prov. La Altagracia, San Rafael del Yuma, La Gran Chorra. 11.IV.1992, F. Del Monte. 5 q MNHNSD 09.144, prov. Valverde, Mao, Gurabo. 5.VII.1980, B. Reynoso. 2 9 MNHNSD 09.145, prov. La Altagracia, San Rafael del Yuma, Boca de Yuma. 8.V.1988, K. Guerrero, J. Infante. 1 수 $1 \delta^{\Uparrow}$ MNHNSD 09.146, prov. La Altagracia, San Rafael del Yuma, Boca de Yuma. 1.III.1992, 20 우 MNHNSD 09.147, prov. La Romana, Isla Saona, Mano Juan. 26.I.1980, E. Marcano. 1 ㅇ MNHNSD 09.148, prov. San Juan, Vallejuelo. 28.XII.1979, E. Marcano. 4 MNHNSD 09.150, prov. Valverde, Mao, Ámina, Entrada de Mao, Río Guanajuma. 9.VIII.1980, E. Marcano. 49 MNHNSD 09.151, prov. Santiago Rodríguez, San Ignacio de Sabaneta, Zamba. 2.VIII.1980, E. Marcano. 1 ㅇ MNHNSD 09.152, prov. Valverde, Mao, Los Quemados, El Cercado. 2.I.1982, E. Marcano. 2 + MNHNSD 09.153, prov. Santiago Rodríguez, San Ignacio de Sabaneta, Los Ingenitos. 6.VII.1980, E. Marcano. 2 q MNHNSD 09.155, prov. Peravia, Baní, Galeón. 19.X.1980, J. Cicero, E. Marcano. 82 ㅇ MNHNSD 09.156, prov. Azua, Tábara Arriba, Tábara Abajo. 22.XII.1979, E.Marcano. $2{ }^{\dagger}$ MNHNSD 09.157, prov. Azua, Tábara Arriba, Tábara Abajo. 22.XII.1979, E. Marcano. 3 + MNHNSD 09.158, prov. La Altagracia, Higüey, El Salado, Verón. 24.VII.1980, E. Marcano. 2 + MNHNSD 09.159, prov. La Romana, Isla Saona, 1.5km N Mano Juan. 27.I.1980, J. A. Ottenwalder, S. Incháustegui. 2 + MNHNSD 09.160, prov. Santo Domingo, Boca Chica, La Malena. $18^{\circ} 25.461^{\prime} N 6^{\circ} 33.468^{\prime}$ W. 21.III.2003, D. Pérez. 1 ㅇ MNHNSD 09.161, prov. Pedernales, Oviedo, Oviedo 
Viejo. 30.XII.1989, R. Briones. 5 9 MNHNSD 09.162, prov. Elías Piña, Pedro Santana, Río Limpio. 25-27.VI.1999, S. H. C. 1 ㅇ MNHNSD 09.163, prov. Distrito Nacional, Santo Domingo. 3.X.1976, José Gómez. 3 ㅇ 1ð̂ MNHNSD 09.164, prov. Azua. 31.V.1988, J. Infante, S. Navarro. 4ㅇ MNHNSD 09.165, prov. Santiago Rodríguez, San Ignacio de Sabaneta, Las Caobas, Río Gurabo. 1.XII.1979, E. Marcano, J. Cicero. 3 q 1 juv. MNHNSD 09.166, prov. La Altagracia, Higüey, El Salado, Verón. 17.XI.1979, E. Marcano. 1 ㅇ MNHNSD 09.167, prov. Montecristi, El Rincón. 4.V.1980, J. Cicero, E. Marcano. 5 q MNHNSD 09.168, prov. Valverde, Guatapanal, Potrero, Río Ámina. 5.IV.1980. E. Marcano F. 1 q MNHNSD 09.170, prov. Distrito Nacional, Santo Domingo. 16.X.1976, José Gómez. 17ㅇ MNHNSD 09.172, prov. Valverde, Mao, Gurabo Adentro, Río Gurabo. 5.I.1980, E. Marcano. 4 ㅇ MNHNSD 09.173, prov. Azua, Peralta, Majagual, El Puerto. 10.XI.1979, E. Marcano, J. Cicero, A. Abud. 19 MNHNSD 09.174, prov. Elías Piña, Comendador, Cañada Miguel. 27.XII.1979, E. Marcano. 1 ㅇ MNHNSD 09.175, prov. Peravia, Nizao, Nizao. 30.XII.1979, K. Guerrero. 1 ㅇ MNHNSD 09.176, prov. La Romana, Isla Saona, Catuano. 27.I.1980, E. Marcano. 1 ㅇ MNHNSD 09.179, prov. Monseñor Nouel, Bonao, Arroyo Toro, Piedra Gorda, Arroyo Piedra Gorda, Charco Bonito. 5.XI.1988, F. Del Monte. 19 MNHNSD 09.177, prov. La Vega, Constanza, El Arroyazo. 18.IX.1999, D. Pérez, R. Bastardo, S. Medrano. $2 \widehat{\jmath}$ MNHNSD 09.178, prov. La Romana, carretera La Romana-Higüey, represa L. Charón. 9.V.1991, K. Guerrero. 1 9 MNHNSD 09.207, prov. Santiago Rodríguez, San Ignacio de Sabaneta, Las Caobas. 5.VII.1980, B. Reynoso. 2 q MNHNSD 09.182, prov. Montecriti, Guayibín, La Solitaria. 18.I.1981, E. Marcano. 7 우 MNHNSD 09.183, prov. Azua, Tábara Arriba, Tábara Abajo. Río Arroyo Blanco. 26.XII.1979. E. Marcano. 1 우 MNHNSD 09.185, Monseñor Nouel, Bonao, Casabito. 25.VIII.1979, E. Marcano. 3 9 MNHNSD 09.186, prov. Distrito Nacional, Santo Domingo de Guzmán. 16.X.1976, José Gómez. 3 9 MNHNSD 09.187, prov. Distrito Nacional, Santo Domingo de Guzmán. 16.X.1976, Rosemary Gómez. 1우 MNHNSD 09.188, prov. Santiago, Tamboril, Guazumal Arriba. 4.XI.1979, E. Marcano. 5ㅇ MNHNSD 09.189, prov. Azua, Cortez. 24.V.1981, E. Marcano. 1우 MNHNSD 09.191, prov. Bahoruco, Neiba. 25.X.1980, E. Marcano. 1 ㅇ MNHNSD 09.192, prov. Valverde, Mao, Los Quemados, El Cercado. 4.IV.1980, E. Marcano. 1 + MNHNSD 09.193, prov. La Altagracia, San Rafael del Yuma, Boca de Yuma.

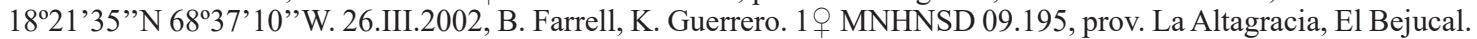
21.I.1992, K. Guerrero, León. 1 우 MNHNSD 09.196, prov. La Altagracia, Higüey, Jina Jaragua, Punta Cana. 22-31. III.2002, B. Farrell, K. Guerrero. 1 q MNHNSD 09.198, prov. La Romana, Isla Saona. 23.VI.1992, G. V. 1 q MNHNSD 09.199, prov. La Altagracia, San Rafael del Yuma, Benerito. 25.X.1998, S. Medrano. 1ㅇ MNHNSD 09.200, prov. Valverde, Guatapanal, Potrero, Río Ámina. 4.V.1980, E. Marcano. 1 MNHNSD 09.206, prov. Independencia, La Descubierta, Los Borbollones. 2050511N 211239E. 5.V.2006, S. Medrano, M. Sánchez, A. Schubert. 1 ㅇ MNHNSD 09.1182, prov. Santo Domingo, Santo Domingo Oeste, Engombe. 21.XI.2007, curso UASD.

Gasteracantha sp. (1 lote, 1 espécimen)

1 juv. MNHNSD 09.208, Puerto Rico, Puente Blanco, Quebradillas. 4.V.1993.

Larinia Simon, 1874

Larinia minor (Bryant, 1945) (1 lote, 1 espécimen)

1 우 MNHNSD 09.209, prov. Barahona, Paraíso, Los Patos, playa Los Patos. 31.VIII.1976, J. A. Ottenwalder.

Mecynogea Simon, 1903

Mecynogea sp. (3 lotes, 7 especímenes)

1 우 MNHNSD 09.210, prov. La Altagracia. $18^{\circ} \mathrm{N} 68^{\circ} \mathrm{W}$. 22-31.III.2002, B. Farrell, K. Guerrero. 1 juv. MNHNSD 09.211, prov. La Altagracia, San Rafael del Yuma, La Gran Chorra. 11.IV.1992, F. Del Monte. 1 + 4 juv. MNHNSD 09.212, prov. Independencia, La Descubierta, Isla Cabritos. 18.VI.1981, E. Marcano.

\section{Metazygia Cambridge, 1903}

Metazygia crewi (Banks, 1903) (3 lotes, 6 especímenes)

1 q MNHNSD 09.213, prov. Peravia, Baní, Honduras, El Matadero. 25-26.X.2008, V. De la Rosa, A. Hilario, G. de los Santos. 1 juv. MNHNSD 09.214, prov. Independencia, La Descubierta, Los Borbollones. 30.I.1991, F. Del Monte. 4 + MNHNSD 09.215, prov. La Altagracia, San Rafael del Yuma, La Gran Chorra. 11.IV.1992, F. Del Monte.

Metazygia dubia (Keyserling, 1864) (3 lotes, 16 especímenes)

5 ㅇ 5 juv. MNHNSD 09.216, prov. Bahoruco, terreno salado entre Neiba y Duvergé. 25.X.1980, E. Marcano. 4 우 MNHNSD 09.217, prov. Santo Domingo, Santo Domingo Este, Acuario Nacional. 13.VIII.1991, F. Del Monte. 29 MNHNSD 09.218, prov. Samaná, Las Terrenas. 26.XII.1992, F. Del Monte.

Metazygia genialis (Keyserling, 1892) (1 lote, 4 especímenes)

$2 \widehat{\jmath} 1$ 우 1 juv. MNHNSD 09.220, prov. La Vega, cruce de Jima Abajo. 25.XII.1992, F. Del Monte.

Metazygia gregalis (O. P.-Cambridge, 1889) (3 lotes, 7 especímenes)

1 ㅇ 1 juv. MNHNSD 09.219, Panamá, prov. Chiriquí, David, Centro Regional Universitario. 26.XI.1975, D. Quintero. 4우 MNHNSD 09.220, prov. La Vega, Jima Abajo. 25.XII.1992, F. Del Monte. 1 ㅇ MNHNSD 09.221, prov. Barahona, Jaquimeyes, Puerto Alejandro.26.X.1980, E. Marcano. 
Metazygia pallidula (Keyserling, 1864) (1 lote, 2 especímenes)

$1{ }^{\circledR} 1$ ㅇ MNHNSD 09.222, Panamá, prov. Chiriquí, Boquete. VII.1939. A. M. Chickering.

Metazygia sp. (4 lotes, 10 especímenes)

4 ㅇ 2 juv. MNHNSD 09.34, prov. Azua, Las Yayitas. 15.I.2002, S. Medrano.1 juv. MNHNSD 09.223, prov. Pedernales, La Agüita, $1 \mathrm{~km}$ antes del cruce de Banano. 14.VIII.1991, K. Guerrero, D. Matusik. 2 ㅇ MNHNSD 09.338, prov. La Altagracia, San Rafael del Yuma. 30.V.1992. 1 우 MNHNSD 09.1197, prov. Independencia, Duvergé, La Zurza, $18^{\circ} 24^{\prime} 25.1^{\prime \prime}$ N 71³4'25.4’W. 5.IX.2009, G. de los Santos.

Metepeira Cambridge, 1903

Metepeira datona Chamberlin \& Ivie, 1942 (2 lotes, 8 especímenes)

$2 q$ MNHNSD 09.225, prov. La Romana, Isla Saona. 24.VII.1992, F. Del Monte, K. Guerrero. 69 MNHNSD 09.226, prov. Barahona, Paraíso, Los Patos, playa Los Patos. 31.VIII.1976, J. A. Ottenwalder.

Metepeira triangularis (Franganillo, 1930) (6 lotes, 39 especímenes)

5 ㅇ MNHNSD 09.227, prov. Azua, Las Charcas, Hatillo. 30.I.1991, F. Del Monte. 3 9 MNHNSD 09.228, prov. Montecristi, carretera Montecristi-El Morro. 21.XII.1991, F. Del Monte. 19 MNHNSD 09.229, prov. Independencia, Jimaní, 2km SE El Limón. 18.25N 71.45W. 30.I.1991, F. Del Monte. 3 ํ 10 MNHNSD 09.230, prov. Peravia, Baní, Las Calderas, Las Dunas. 23.I.1992, F. Del Monte. 24우 MNHNSD 09.231, prov. Independencia, La Descubierta, Isla Cabritos. 18.VI.1981, E. Marcano. 2 ㅇ MNHNSD 09.232, prov. Montecristi, carretera Montecristi-El Morro. 21.XII.1991, F. Del Monte.

Metepeira vigilax (Keyserling, 1893) (1 lote, 1 espécimen)

1 ㅇ MNHNSD 09.1195, prov. Azua, Peralta, Majagual, El Puerto. 10.XI.1979, E. Marcano.

Metepeira sp. (13 lotes, 40 especímenes)

2 q MNHNSD 09.233, prov. Pedernales, Pedernales, Cabo Rojo. 20.V.1992. 1 + MNHNSD 09.235, prov. La Vega, Constanza, El Convento, Cruce de Zocaba. 13.VII.1998, S. Navarro, K. Polanco, S. Medrano. 193 juv. MNHNSD 09.237, prov. Peravia, Baní, Galeón. 22.VIII.1979, E. Marcano. $10^{\Uparrow} 12$ 2 2 juv. MNHNSD 09.238, prov. Independencia, La Descubierta, Isla Cabritos. 4.VII.1981, E. Marcano. 6910 T MNHNSD 09.240, prov. La Altagracia, San Rafael del Yuma, 4-5km S caseta La Gran Chorra. 1.V.1992. 1 juv. MNHNSD 09.239, Puerto Rico, Puente Blanco, Quebradillas. 3 MNHNSD 09.241, prov. Peravia, Baní, Las Calderas, Las Salinas. 2.XII.2007, A. Sánchez. 1 ㅇ MNHNSD 09.242, prov. La Altagracia, Higüey, Jina Jaragua, Punta Cana. $18^{\circ} 30^{\prime} 59^{\prime}$ 'N 68 22'31'”W. 22-31.III.2002, B. Farrell, K.

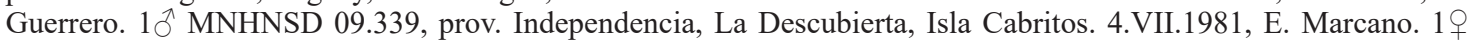
1 juv. MNHNSD 09.1193, prov. La Vega, Constanza, Cuevita, $18^{\circ} 44.469^{\prime}$ N 7040.227'W. 28.XI.2002, D. Perez, B. Hierro, R. Bastardo. 1 q MNHNSD 09.1194, prov. La Altagracia, San Rafael del Yuma. 30.V.1992. 1 \% MNHNSD 09.381, prov. Pedernales, Oviedo, $17^{\circ} 54.901^{\prime} \mathrm{N} 71^{\circ} 30.067^{\prime}$ W. 4.VII.2002, D. Perez, R. Bastardo, B. Hierro. 1 ㅇ MNHNSD 09.1196, prov. La Romana, Isla Saona.

\section{Micrathena Sundevall, 1833}

Micrathena forcipata (Thorell, 1859) (4 lotes, 8 especímenes)

1 ㅇ MNHNSD 09.243, prov. La Romana, Isla Saona, km 51ำ camino Mano Juan-Catuano. 18.VII.1974, J. Alberto Ottenwalder. 4 9 1 juv. MNHNSD 09.244, prov. La Romana, Isla Saona. 23.VI.1992, G. V. 1 juv. MNHNSD 09.283, prov. Pedernales, La Agüita, $1 \mathrm{~km}$ antes del cruce de Banano. 14.VIII.1991, K. Guerrero, D. Matusik. 19 MNHNSD 09.281, prov. La Romana, Isla Saona.

Micrathena militaris (Fabricius, 1775) (21 lotes, 44 especímenes)

1 ㅇ MNHNSD 09.245, prov. La Vega, Jarabacoa, Paso Bajito, La Sal. 1904.101'N 70³4.089'W. 11-12.VII.2002. D. Pérez, B. Hierro, R. Bastardo. 1 q MNHNSD 09.247, prov. Samaná. 6.XII.2007, H. Andújar. 1 9 MNHNSD 09.248, prov. La Vega, Constanza, El Arroyazo. $1^{\circ} 1.995^{\prime}$ N 70³2.593'W. 9-10.VII.2002. 1 ㅇ MNHNSD 09.249, prov. San Cristóbal, Cambita-Garabitos, Majagual. 12.IV.1976. Gómez-Marión. 1q MNHNSD 09.250, prov. Pedernales, Hoyo de Pelempito.10.XII.1978, D. G: Robinson. 1 ㅇ MNHNSD 09.251, prov. San Juan, El Cercado, La Hermita. 28.III.1981, E. Marcano. 491 juv. MNHNSD 09.252, prov. Elías Piña, Comendador, Cañada Miguel. 27.XII.1979, E. Marcano. 2 9 MNHNSD 09.253, prov. Hato Mayor, Sabana de la Mar, La Cruz, puente sobre Río Yabón. 12.I.1980, E. Marcano. 1ㅇ MNHNSD 09.255, prov. Monseñor Nouel, Bonao. 17.X.1976, Leo Salazar. 1 ㅇ MNHNSD 09.257, prov. La Altagracia, San Rafael del Yuma, Boca de Yuma. 8.V.1988, K. Guerrero, J. Infante. 1 ㅇ MNHNSD 09.258, prov. La Romana, Guaymate, La Noria. 4.XI.1989, K. Guerrero. 1 + MNHNSD 09.259, prov. Monseñor Nouel, Bonao, Charco Bonito, Arroyo Piedra Gorda. 5.XI.1988, F. Del Monte. 1 + MNHNSD 09.261, prov. La Vega, Jarabacoa, detrás del Hotel Montaña. 2.VI.1993, K. Guerrero. 1 9 MNHNSD 09.262, prov. Duarte, San Francisco de Macorís, Loma Quita Espuela. 24.X.1976, J: A. Ottenwalder. 1 \% MNHNSD 09.264, prov. La Vega, Jarabacoa, Paso de la Perra. $19^{\circ} 04.576$ ’N 70 49.623’W. 16.VII.2002, D. Pérez, B. Hierro, R. Bastardo, S. Medrano, H. Takizawa. 39 MNHNSD 09.265, prov. La Romana, Isla Saona, Catuano. 27.I.1980, E. Marcano. 3 q MNHNSD 09.267, prov. Elías Piña, Pedro 
Santana, Río Limpio. 23-27.VI.1999. 19 MNHNSD 09.268, prov. La Vega, Jarabacoa, Los Tablones. 21.VII.2002, S. Medrano. 9 ㅇ MNHNSD 09.269, prov. Santiago, San José de las Matas, El Limón. 1.II.1981, E. Marcano. 3 ㅇ MNHNSD 09.270, prov. La Altagracia, Higüey, El Salado, Verón. 24.VII.1982, E. Marcano. 4 \& 1 juv. MNHNSD 09.275, prov. Santo Domingo, Santo Domingo Oeste, Engombe. 21.X.2007, curso UASD.

Micrathena similis Bryant, 1945 (5 lotes, 10 especímenes)

3 은 MNHSD 09.273, prov. La Altagracia, San Rafael del Yuma, Guaraguao. 29-31.V.1992, K. Guerrero. 19 MNHNSD 09.274, prov. Santiago, San José de las Matas, Mata Grande. 21.IV.1999, R. Bastardo. 1 ㅇ MNHNSD 09.278, prov. La Vega, Jarabacoa, Los Tablones. 20.VII.2002, D. Pérez, B. Hierro, R. Bastardo, S. Medrano, H. Takisawa. 2 juv. MNHNSD 09.277, prov. Santiago, San José de las Matas, Diferencia. $1^{\circ} 16.313^{\prime} \mathrm{N} 71^{\circ} 03.132^{\prime} \mathrm{W}$. 8.IV.2003, D. Pérez, B. Hierro, R. Bastardo. 3 9 MNHNSD 09.1198, prov. Peravia, Baní, Honduras, Los Cedros, UTM 19Q 351473m.E 2036495m.N. 23.X.2009, G. de los Santos.

Micrathena sp. (13 lotes, 40 especímenes)

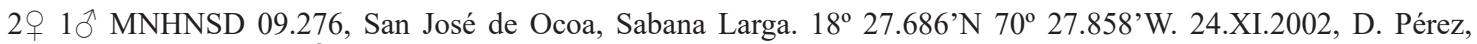
B.Hierro, H. Andújar. 19 MNHNSD 09.279, prov. La Vega, Jarabacoa, Los Tablones. $1^{\circ} 03.284^{\prime} \mathrm{N} 70^{\circ} 53.227^{\prime} \mathrm{W}$. 4.IX.2001, S. Medrano. 1 우 MNHNSD 09.280, prov. Monseñor Nouel, Bonao, Blanco, El Pichón. 27-30.VI.1998, D. Veloz, S. Navarro. $1+$ MNHNSD 09.282, prov. Elías Piña, Pedro Santana, Río Limpio. 25-27.VI.1999, S. Medrano.

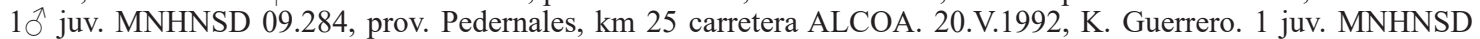
09.1199, prov. San Cristóbal, Cambita-Garabitos, El Majagual, UTM 19Q 371510m.E 2044198m.N. 23.I.2009, C. Suriel, C. Marte, S. Carrero.

\section{Neoscona Simon, 1864}

Neoscona marcanoi Levi, 1993 (6 lotes, 10 especímenes)

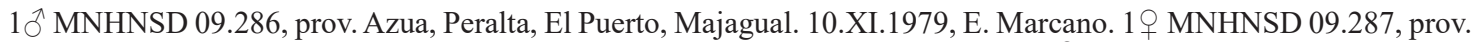
Azua, Tábara Arriba, Tábara Abajo, Río Arroyo Blanco. 22.XII.1979, E. Marcano. 1 q MNHNSD 09.288, prov. Azua, Quitacoraza. 8.XII.1991, K. Guerrero. 4910 MNHNSD 09.289, prov. Independencia, La Descubierta, Isla Cabritos. 1.VIII.1981, E. Marcano. 1 9 MNHNSD 09.290, prov. Independencia, La Descubierta, Isla Cabritos. 28.V.1981, P. Merejo. 1우 MNHNSD 09.291, prov. Puerto Plata, Altamira, La Cumbre. 3.IX.1979, E. Marcano.

Neoscona nautica (L. Koch, 1875) (2 lotes, 4 especímenes)

1 MNHNSD 09.292, prov. La Vega, Constanza. XI.1991, F. Del Monte. 3 q MNHNSD 09.293, San Pedro de Macorís, San Pedro de Macorís, Guayacanes. 25.I.1980, J. Cicero.

Neoscona sp. (31 lotes, 62 especímenes)

1ðิ MNHNSD 09.294, Peravia, Baní, Honduras, El Matadero. 25-26.X.2008. V. De la Rosa, A. Hilario, G. de los Santos. 1 q MNHNSD 09.296, prov. Elías Piña, Pedro Santana, Río Limpio. 25-27.VI.1999. 3 juv. MNHNSD 09.297, prov. Elías Piña, Comendador, Cañada Miguel. 27.XII.1979, E. Marcano. 2 juv. MNHNSD 09.298, prov. Elías Piña, Pedro Santana, Guayajayuco, Río Artibonito. 9.XI.1980, E. Marcano. 1 MNHNSD 09.299, prov. Monseñor Nouel, Bonao, Tireito. 25.V.1998. 2 ㅇ 1 juv. MNHNSD 09.300, prov. Distrito Nacional, Santo Domingo, Arroyo Salado. 25.VIII.1976, J. A. Ottenwalder, D. G. Robinson. 1 ㅇ MNHNSD 09.302, prov. Santo Domingo, Los Alcarrizos, Savica, Los Alcarrizos III. 9.IX.1994, S. Marte. 1ㅇ MNHNSD 09.303, prov. Santo Domingo, Los Alcarrizos, Savica, Los Alcarrizos III. 9.IX.1994, S. Marte. 3 juv. MNHNSD 09.304, prov. Pedernales, Las Abejas, Sierra de Bahoruco. 19.VI.1991, D. Matusik, K. Guerrero. 1 juv. MNHNSD 09.305, prov. Independencia, La Descubierta, Isla Cabritos. 1.VI.1981, R. Briones, M. Silva. 1 9 MNHNSD 09.341, prov. Independencia, La Descubierta. 4.VII.1981, E. Marcano. 291 juv. MNHNSD 09.400, prov. La Vega, Jarabacoa, Manabao, 194.576'N 7049.623'W. 19.VII.2002, S. Medrano. 4 ㅇ 1 juv. MNHNSD 09.373, prov. La Vega, Constanza, Cuevita, 1844.469'N 70²0.227’W. 28.XI.2002, D. Perez, B. Hierro, R. Bastardo. 10 MNHNSD 09.361, prov. La Vega, Constanza, El Arroyazo. 18.IX.1999, D. Perez, R. Bastardo, S. Medrano. $1 \delta^{\top} 1$ ㅇ MNHNSD 09.337, prov. La Vega, Constanza, 1901.957’ N 70²9.124'W. 31.VII.2001, S. Medrano. 1 ㅇ MNHNSD 09.355, prov. Santiago, San José de las Matas, El Limón. 1.II.1981, E. Marcano. 10 MNHNSD 09.335, prov. Monseñor Nouel, Bonao, Blanco, El Pichón. 27-30.V.1998, D. Veloz, S. Navarro. 1 ㅇ 2 juv. MNHNSD 09.1200, prov. San Juan, Sabaneta, Alto de la Rosa. 8.I.2009, A. Sánchez, R. Rodríguez. 1 우 $1 \delta^{\lambda}$ MNHNSD 09.372, prov. Monseñor Nouel, Bonao, Tireito. 25.V.1998. 1 우 MNHNSD 09.378, prov. Distrito Nacional, Santo Domingo. 16.X.1976, Rosse Mary Gómez. 19 MNHNSD 09.376, prov. Montecristi, 1948.933’N

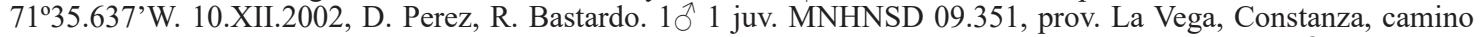
El Arroyazo-La Sal, $1^{\circ} 02.374^{\prime}$ N 70³2.648'W. 10.VII.2002, D. Perez, R. Bastardo, B. Hierro. 19 MNHNSD 09.1201, prov. Independencia, La Descubierta, Isla Cabritos. 18.VI.1981, E. Marcano. 1 q 4 juv. MNHNSD 09.1202, prov. Independencia, La Descubierta, La Azufrada, 18³3'46.8”N 7141'52.0"W. 10.X.2009, G. de los Santos.

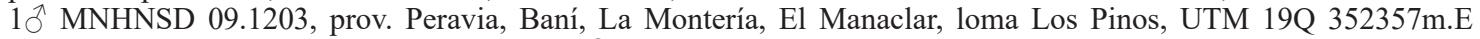
2035657m.N. 26.IX.2009, G. de los Santos. 1 오 MNHNSD 09.1204, prov. Peravia, Baní, La Montería, El Manaclar, transepto loma Los Guayuyos-Segungdo río, UTM 19Q 354040m.E 2036965m.N. 29.VIII.2009, G. de los Santos. 2 PNHNSD 09.1205, prov. Peravia, Baní, La Montería, El Manaclar, transepto loma Los Guayuyos-Segungdo río, UTM 19Q 354040m.E 2036965m.N. 29.VIII.2009, G. de los Santos. $1{ }^{\lambda}$ MNHNSD 09.1206, prov. Peravia, Baní, La Montería, El Manaclar, loma Los Guayuyos, UTM 19Q 353421m.E 2036933m.N. 25.IX.2009, G. de los Santos. $1+$ MNHNSD 09.1215, prov. Monseñor Nouel, Bonao, Tireito. 25.V.1998. 1 đ̄ 7 juv. MNHNSD 09.234, prov. Pedernales, 
Aceitillar, Sierra de Bahoruco. $18^{\circ} 04.44^{\prime} N$ 71 $38.391^{\prime}$ W. 2.IX.2001, S. Medrano. 29 MNHNSD 09.236, prov. Pedernales, Los Güiritos, Sierra de Bahoruco. $18^{\circ} 09.178^{\prime}$ N 71 35.470’W. 2.IX.2001, K. Guerrero.

Ocrepeira Marx, 1883

Ocrepeira darlingtoni (Bryant, 1945) (1 lote, 1 espécimen)

1 우 MNHNSD 09.306, prov. Pedernales, La Agüita, Cruce de Banano. 14.VIII.1991, K. Guerrero, D. Matusik.

Pozonia Schenkel, 1953

Pozonia sp. (1 lote, 1 espécimen)

1ठ઼ MNHNSD 09.336, prov. La Altagracia, San Rafael del Yuma. 6.V.1988, J. Infante.

Verrucosa Mello-Leitão, 1939

Verrucosa arenata (Walckenaer, 1842) (14 lotes, 21 especímenes)

1 9 MNHNSD 09.307, prov. Barahona, Paraíso, Paraíso. 22.VI.1980, E. Marcano. 1q MNHNSD 09.308, prov. Duarte, San Francisco de Macorís, La Maníigua. 2.VII.1976, J. A. Ottenwalder, S. Incháustegui. 19 MNHNSD 09.309, prov. Samaná, Sánchez, Los Naranjos Arriba. 20.I.1980, J. A. Ottenwalder. 1 + MNHNSD 09.310, prov. Barahona, Polo, Monteada Nueva. 24.VII.1977, J. A. Ottenwalder. 1 NNNSD 09.312, prov. Elías Piña, Pedro Santana, Río Limpio. 22-25.VI.1999. 191 juv. MNHNSD 09.314, prov. Elías Piña, Pedro Santana, Río Limpio. 12.IX.1999. 29 MNHNSD 09.315, prov. El Seibo, El Seibo, Loma La Herradura. 23.II.1991, G. Dominici. 2 q 2 juv. MNHNSD 09.316, prov. Samaná, Sánchez, El Naranjo. 18.I.1980, E. Marcano. 2 q MNHNSD 09.317, prov.Pedernales, Pedernales, Las Mercedes, carretera ALCOA. 24.I.1981, J. A. Ottenwalder, S. Incháustegui, N. G. M., D. G. 1 9 MNHNSD 09.318, prov. Independencia, Postrer Río. $18^{\circ} 39.339^{\prime} N$ 71 39.279’W. 27.III.2003, D. Pérez, R. Bastardo, B. Hierro. 1 ㅇ 1 juv. MNHNSD 09.319, prov. La Romana, Isla Saona, Mano Juan. 25.VII.1992, F. Del Monte, K. Guerrero. 1 q MNHNSD 09.320, prov. La Altagracia, San Rafael del Yuma, PN del Este. 8.III.1994. 1 q MNHNSD 09.321, prov. Pedernales, carretera ALCOA. 20.V.1992. 1 + MNHNSD 09.322, prov. La Vega, Constanza, Valle Nuevo. 25.V.1998.

Verrucosa sp. (4 lotes, 7 especímenes)

1 ㅇ MNHNSD 09.323, prov. La Vega, Jarabacoa, Paso Bajito, La Sal. $19^{\circ} 04.101^{\prime}$ N 70 34.088'W. 11-12.VII.2002, D. Pérez, B. Hierro, R. Bastardo. 19 MNHNSD 09.324, prov. La Vega, Constanza, Las Palomas, El Arroyazo. $1^{\circ}$ $1.995^{\prime} \mathrm{N} 70^{\circ} 32.593$ 'W. 9-10.VII.2002. 4ㅇ MNHNSD 09.325, prov. La Altagracia, San Rafael del Yuma, Bayahibe. 25.I.1980, E. Marcano. 19 MNHNSD 09.326, prov. Elías Piña. $1^{\circ} 14.908^{\prime}$ N 71º 32.228’W. 25.VII.2003, D. Pérez, B. Hierro, R. Bastardo.

Wagneriana Cambridge, 1904

Wagneriana vegas Levi, 1991 (1 lote, 1 espécimen)

PARATIPO: 1 ㅇ MNHNSD 09.327, prov. La Romana, Isla Saona, Catuano. 27.I.1980, E. Marcano.

Wagneriana sp. (1 lote, 1 espécimen)

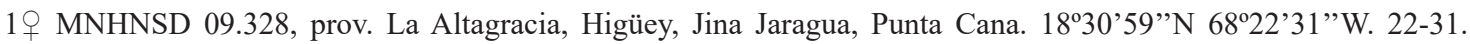
III.2002, B. Farrell, K. Guerrero.

Witica Cambridge, 1895

Witica crassicaudus (Keyserling, 1865) (2 lotes, 2 especímenes)

1 juv. MNHNSD 09.329, prov. Hato Mayor, Hato Mayor, Manchado, Arroyo Guamira. 9.V.1991, F. Del Monte, K. Guerrero. $1+$ MNHNSD 09.330, prov. La Romana, Isla Saona. 23.VI.1992, G. V. 